\title{
CRESCIMENTO INICIAL DE Dalbergia nigra (Vell.) Allemão ex. Benth. (FABACEAE) E Chorisia speciosa A.St.-Hil (MALVACEAE) SOB DIFERENTES NÍVEIS DE SOMBREAMENTO ${ }^{1}$
}

\author{
Fernanda Ventorim Pacheco ${ }^{2}$, Carlos Rodrigues Pereira ${ }^{3}$, Rogério Luiz da Silva ${ }^{4}$ e Ivan Caldeira Almeida \\ Alvarenga ${ }^{5}$
}

\begin{abstract}
RESUMO - A produção de mudas florestais, seja com finalidade econômica ou conservacionista, depende de conhecimentos relacionados ao crescimento e desenvolvimento vegetal. O estudo de uma planta em diferentes condições ambientais permite definir e aprimorar formas de cultivos mais adequadas. No trabalho, objetivou-se avaliar o crescimento de mudas de Dalbergia nigra (jacarandá caviúna) e Chorisia speciosa (paineira) cultivadas em casas de vegetação sob diferentes níveis de sombreamento, com o intuito de determinar o sombreamento mais adequado ao crescimento e relacionar suas respostas ao contexto ecológico. As sementes das espécies foram germinadas e cultivadas em casas de vegetação nas condições de 0, 22, 50, 70, 84 e 91 \% de sombreamento. As variáveis analisadas foram altura de plantas, diâmetro do colo, área foliar total e a relação altura/diâmetro, sendo coletados os dados aos 30, 60, 90 e 180 dias após a emergência. As mudas de jacarandá caviúna tiveram maior altura, diâmetro do colo e área foliar nos tratamentos com sombreamento intermediários (50\%, 70\% e $84 \%$ de sombra) e apresentou características de espécies tolerantes a sombra e de estágios mais avançados de sucessão, se aproximando de espécies secundárias tardias a clímax. Por outro lado, as mudas de paineira apresentaram melhor crescimento no tratamento sem sombra (0\%) e nos tratamentos com sombreamentos leves (20 e 50\%), com características de espécies heliófitas em etapas iniciais de sucessão e aproximando-se de plantas pioneiras a secundárias iniciais.
\end{abstract}

Palavras-chave: Luminosidade; Produção de mudas; Espécies florestais.

\section{INITIAL GROWING OF Dalbergia nigra (Vell.) Allemão ex. Benth. (FABACEAE) AND Chorisia speciosa A.St.-Hil (MALVACEAE) AT DIFFERENT LEVELS OF SHADE}

\begin{abstract}
The seedling production with conservation or economic purpose depends on knowledge related with the growth and development of plants. The study of plants under different environmental conditions, allows to define ways to improve crops. The present study aimed to evaluate the growth of Dalbergia nigra (rosewood caviúna) and Chorisia speciosa (kapok) seedlings cultivated in greenhouses in order to determine the appropriate shading for growing those species and relate their responses to the ecological context. Seeds were sown and grown in greenhouses at 0, 22, 50, 70, 84 and 91\% shading. The growth variables assessed were plant height, stem diameter, leaf area and height/diameter. Data were collected at 30, 60, 90 and 180 days after emergence. Seedlings of Dalbergia nigra had a greater height, stem diameter and leaf area in the treatments with intermediate shading (50\%, 70\% and 84\% shade) and showed characteristics of shade-tolerant species and approaching the later stages of succession to late secondary species climax. Moreover, the seedlings of Chorisia speciosa showed better growth in the treatment without shade (0\%) and light shading treatments (20 and 50\%), with characteristics of species heliophytic plants in the early stages of succession, as pioneer plants or early secondary.
\end{abstract}

Keywords: Luminosity; Seedlings production; Forest species.

\footnotetext{
${ }^{1}$ Recebido em 08.12.2012 aceito para publicação em 27.08.2013.

${ }^{2}$ Programa de Pós-Graduação em Fisiologia Vegetal na Universidade Federal de Lavras, MG, Brasil. E-mail: <fventorimpacheco@yahoo.com.br>.

${ }^{3}$ Departamento de Engenharia Agrícola e do Meio Ambiente da Universidade Federal Fluminense, RJ, Brasil. E-mail: <carlosrodriguespereira@yahoo.com.br>.

${ }^{4}$ Universidade Federal Rural do Rio de Janeiro, Instituto de Florestas, RJ, Brasil. E-mail: <rogeriosilva@ufrrj.br>.

${ }^{5}$ Programa de Pós-Graduação em em Fitotecnia na Universidade Federal de Lavras, UFLA, Brasil. E-mail: <pytchouai@yahoo.com.br>
} 


\section{INTRODUÇÃO}

As formações florestais do Brasil vêm sofrendo níveis significativos de perturbação devido ao avanço das fronteiras agropecuárias, das atividades madeireiras, da construção de usinas hidrelétricas e à especulação imobiliária. Nesse cenário, vem crescendo as iniciativas para a execução de projetos de conservação, recuperação e uso sustentável dessas áreas, principalmente com o uso de espécies nativas. Em virtude da carência de conhecimentos sobre a produção de mudas, seu comportamento ecológico e silviculturais em diferentes condições ambientais, são de extrema importância estudos para o desenvolvimento da atividade florestal e para programas de conservação.

A espécie Dalbergia nigra (Vell.) Allemão ex. Benth. conhecida popularmente como jacarandá-da-bahia, jacarandá-preto ou caviúna, é uma leguminosa de porte arbóreo com ocorrência na Floresta Ombrófila Densa (FlorestaAtlântica)(LORENZI, 2002; CARVALHO, 2003). É conhecida comercialmente há mais de trezentos anos, por ser uma das mais valiosas espécies madeireiras que ocorrem no Brasil. Sua madeira, muito procurada para moveis, foi objeto de exportação desde os tempos coloniais (CARVALHO, 2003). Desta forma, a espécie sofreu um intenso e desordenado processo de exploração extrativista entrando na lista oficial das espécies da flora brasileira ameaçadas de extinção (OLIVEIRA FILHO, 1994). A espécie Chorisia speciosa A. St.-Hil. (Malvaceae) é uma espécie arbórea tropical de grande porte e ampla distribuição geográfica, apresentando várias nomenclaturas populares como paineira, paineira-rosa, paineira-branca, árvore-de-paina, dentre outras. Por se tratar de uma planta com crescimento rápido e com características ornamentais, principalmente na fase de florescimento, é muito utilizada para o paisagismo de áreas publicas (LORENZI, 2002).

Alguns estudos realizados mostraram que diferentes níveis de radiação podem alterar a superfície foliar, a altura e o diâmetro do colo em espécies florestais (OLIVEIRA; PEREZ, 2012; AZEVEDO etal., 2010; ORTEGAet al., 2006; FANTI; PEREZ, 2003). Características tais que podem indicar como as condições de radiação interferem na produção de mudas e no comportamento ecológico dessas espécies (SCALON etal., 2001; CAMPOS; UCHIDA, 2002; JUNIOR et al., 2005; SILVA et al., 2007).

Desenvolveu-se o trabalho com o objetivo de avaliar o crescimento de mudas de Dalbergia nigra (jacarandá caviúna) e Chorisia speciosa (paineira) cultivadas em casas de vegetação sob diferentes níveis de sombreamento, com o intuito de determinar as intensidades de sombreamento mais adequadas ao crescimento e relacionar suas respostas ao contexto ecológico das espécies.

\section{MATERIAL E MÉTODOS}

O experimento foi conduzido na área experimental da Empresa Brasileira de Pesquisas AgropecuáriasEMBRAPA(Centro Nacional de Pesquisa em Agrobiologia), durante o período de março a setembro de 2009, no município de Seropédica, região metropolitana do estado do Rio de Janeiro (2245’18,72" S e 4340’05,31" O).

Os tratamentos foram estabelecidos em casas de vegetação com 5 níveis de sombreamento (22, 50, 70, $84,91 \%)$ mais o tratamento a pleno sol (0\% de sombreamento), totalizando 6 tratamentos. Cada nível de sombreamento foi estabelecido com o uso de sombrites comprados conforme especificações dos fabricantes e ajustados com o uso de um Barra Ceptômetro AccuPAR (Modelo LP PAR 80).

Para a produção das mudas, sementes compradas foram semeadas em sementeiras de areia lavada, com $2 \mathrm{~m}$ de comprimento $\mathrm{x} 1 \mathrm{~m}$ de largura, localizadas dentro de cada nível de sombreamento (0, 22, 50, 70, 84 e 91\%). Foram semeadas 200 sementes por tratamento por espécie as quais foram cobertas por uma fina camada de areia lavada. Ao atingirem $10 \mathrm{~cm}$ de altura, as plântulas foram transplantadas para sacos plásticos com $25 \mathrm{~cm}$ de altura por $20 \mathrm{~cm}$ de diâmetro, contendo substrato formado por argila, areia lavada e esterco bovino curtido em proporções iguais.

Os parâmetros de crescimento medidos foram altura máxima da planta, tomando-se a medida a partir do solo até a gema apical, com auxílio de uma trena graduada; diâmetro do colo, por meio de paquímetro digital (Mitutoyo: Absolute Digimatic) e a área foliar total medida com integrador eletrônico digital (LICOR, Modelo LI-3100C Area Meter). As avaliações foram realizadas aos 30, 60, 90 e 180 dias após emergência (DAE). Também foram registradas as temperaturas máxima, média e mínima e umidade relativa através de estações meteorológicas automáticas, modelo Watch Dog 2550, marca Spectrum Weather ${ }^{\circledR}$, instaladas em cada ambiente de sombra.

As oscilações de temperatura estão representadas na Figura 1, onde se verifica uma maior amplitude térmica 
no tratamento de pleno sol em relação aos demais tratamentos variando entre 21 a $28^{\circ} \mathrm{C}$. A maior temperatura média verificada durante o período de experimento foi $28^{\circ} \mathrm{C}(0 \%)$ e a menor $18,2^{\circ} \mathrm{C}$ no tratamento a $60 \%$ de sombra. A menor amplitude térmica foi observada no tratamento a $84 \%\left(18,6\right.$ a $\left.21,8^{\circ} \mathrm{C}\right)$. A umidade relativa média dos tratamentos variou entre 82 ( $0 \%$ de sombra) e $84,5 \%$ (91\% de sombra).

O delineamento experimental utilizado foi o inteiramente casualizado, sendo avaliadas 24 plantas

Fonte: Estação meteorológica automática instalada em cada ambiente de sombreamento. Source: Authomatic weather station installed in each shading environment.
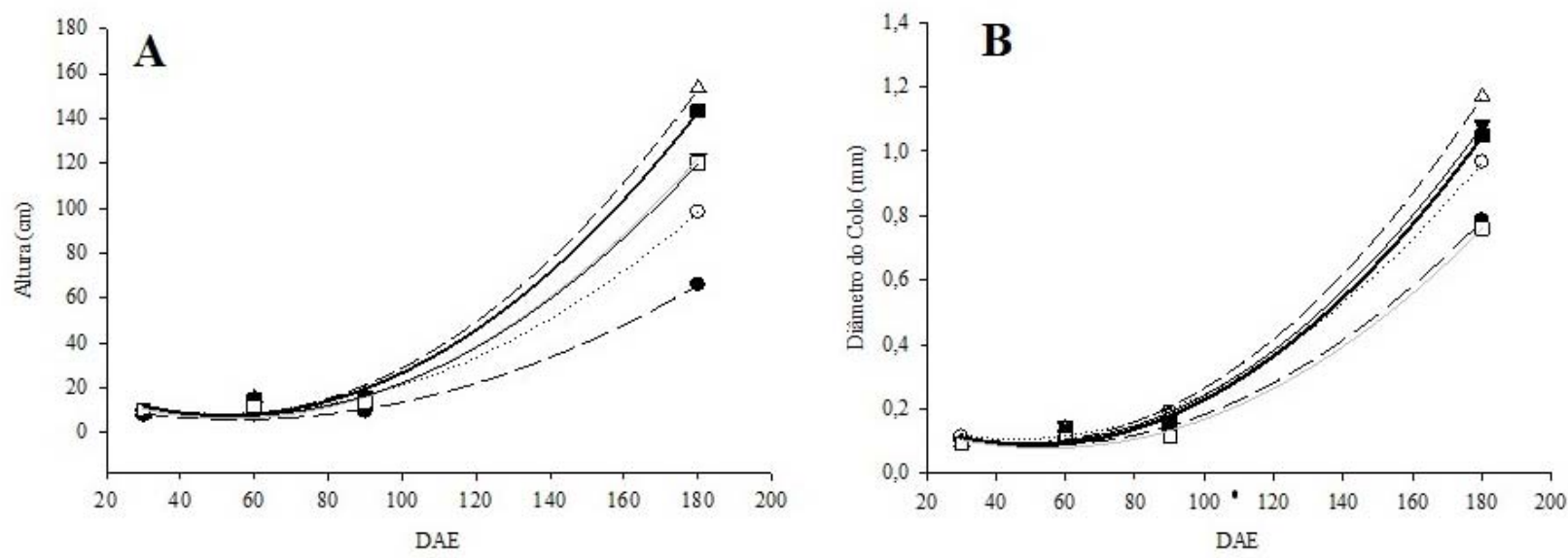

$0 \% \quad \mathrm{Y}=0,0038 \mathrm{x}^{2}-0,4238 \mathrm{x}+17,6121 / \mathrm{R}^{2}=0,9978$

$22 \% \mathrm{Y}=0,0055 \mathrm{x}^{2}-0,5751 \mathrm{x}+23,4365 / \mathrm{R}^{2}=0,9949$

$50 \% \mathrm{Y}=0,0073 \mathrm{x}^{2}-0,7805 \mathrm{x}+27,4061 / \mathrm{R}^{2}=0,9960$

$\triangle 70 \% \mathrm{Y}=0,0087 \mathrm{x}^{2}-0,8794 \mathrm{x}+30,1022 / \mathrm{R}^{2}=0,9950$

- $84 \% \mathrm{Y}=0,0083 \mathrm{x}^{2}-0,8631 \mathrm{x}+30,3226 / \mathrm{R}^{2}=0,994 \mathrm{~S}$

$91 \% \mathrm{Y}=0,0071 \mathrm{x}^{2}-0,7807 \mathrm{x}+28,7987 / \mathrm{R}^{2}=0,9967$
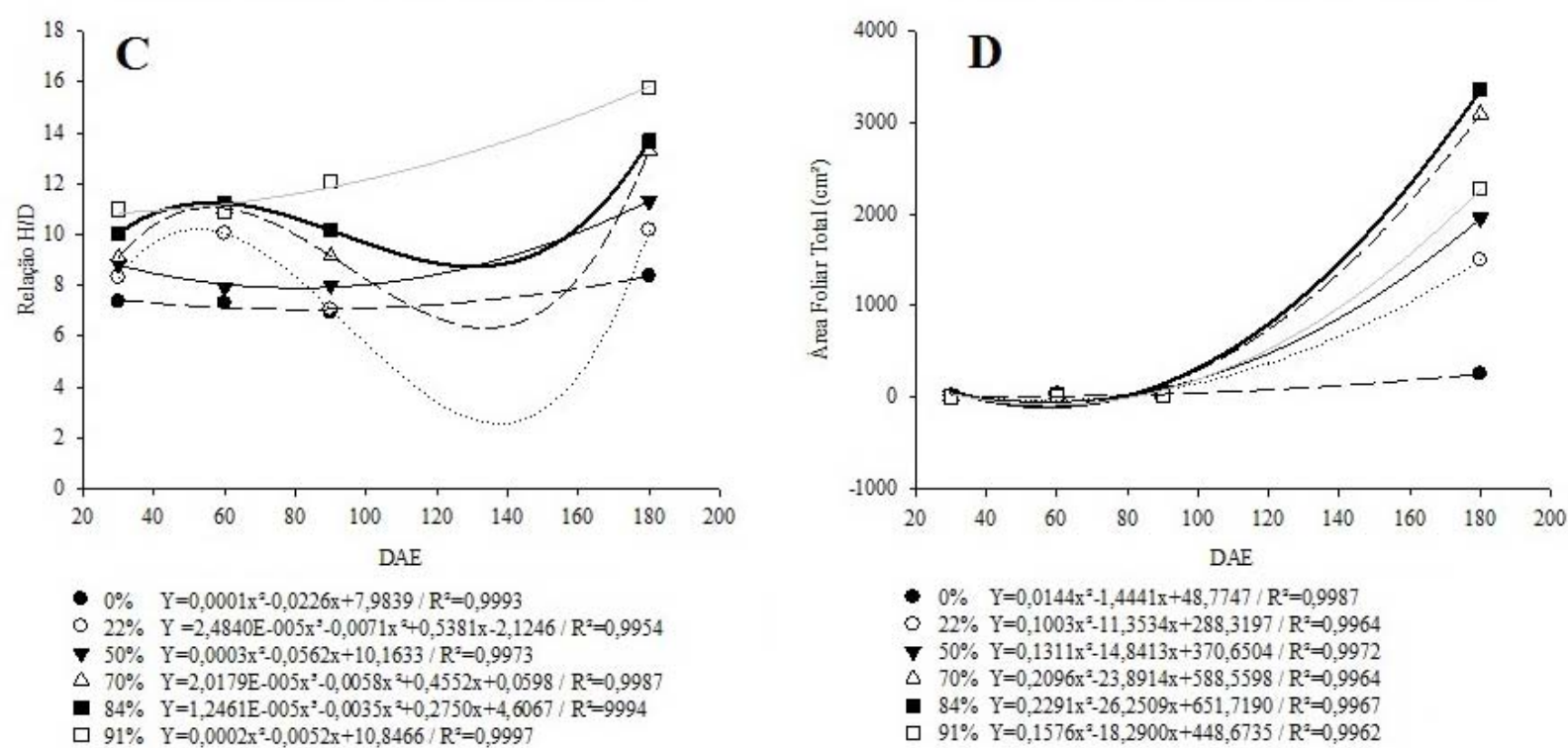

$0 \% \quad \mathrm{Y}=0,0001 \mathrm{x}^{2}-0,0226 \mathrm{x}+7,9839 / \mathrm{R}^{2}=0,9993$

$22 \% \quad \mathrm{Y}=2,4840 \mathrm{E}-005 \mathrm{x}^{2}-0,0071 \mathrm{x}^{2}+0,5381 \mathrm{x}-2,1246 / \mathrm{R}^{2}=0,9954$

- $50 \% \mathrm{Y}=0,0003 \mathrm{x}^{2}-0,0562 \mathrm{x}+10,1633 / \mathrm{R}^{2}=0,9973$

$\triangle 70 \% \mathrm{Y}=2,0179 \mathrm{E}-005 \mathrm{x}^{2}-0,0058 \mathrm{x}^{2}+0,4552 \mathrm{x}+0,0598 / \mathrm{R}^{2}=0,9987$

- $84 \% \quad \mathrm{Y}=1,2461 \mathrm{E}-005 \mathrm{x}^{3}-0,0035 \mathrm{x}^{2}+0,2750 \mathrm{x}+4,6067 / \mathrm{R}^{2}=9994$

ㅁ $91 \% \quad \mathrm{Y}=0,0002 \mathrm{x}^{2}-0,0052 \mathrm{x}+10,8466 / \mathrm{R}^{2}=0,9997$

- $0 \% \quad \mathrm{Y}=4,3366 \mathrm{E}-005 \mathrm{x}^{2}-0,0046 \mathrm{x}+0,2099 / \mathrm{R}^{2}=0,9985$

a $22 \% \quad Y=4,7909 E-005 x^{2}-0,0044 x+0,2083 / R^{2}=0,9995$

- $50 \% \mathrm{Y}=5,7700 \mathrm{E}-005 \mathrm{x}^{2}-0,0057 \mathrm{x}+0,2341 / \mathrm{R}^{2}=0,9952$

$\triangle 70 \% \mathrm{Y}=6,0696 \mathrm{E}-005 \mathrm{x}^{2}-0,0057 \mathrm{x}+0,2277 / \mathrm{R}^{2}=0,9976$

$84 \% \mathrm{Y}=5,6658 \mathrm{E}-005 \mathrm{x}^{2}-0,0057 \mathrm{x}+0,2270 / \mathrm{R}^{2}=0,9962$

ㅁ $91 \% \mathrm{Y}=4,3180 \mathrm{E}-005 \mathrm{x}^{2}-0,0047 \mathrm{x}+0,2066 / \mathrm{R}^{2}=0,9954$

Figura 1 - Oscilação das temperaturas máximas, mínimas e médias nas condições de pleno sol (A), 22\% (B), 50\% (C), 70\% (D), 84\% e 91\% (F) de sombreamento; DAE-Dias após a emergência.

Figure 1 - Oscillation of high, minimal and average in sunny conditions (A); 22\% (B); 50\% (C); 70\% (D); $84 \%$ and 91\% (F) shading; DAE-Days after emergence. 
para cada idade (30, 60, 90 e 180 dias) dentro de cada nível de sombreamento $(0,22,50,70,84$ e 91\%) totalizando 96 plantas para cada espécie.

As médias das variáveis foram comparadas entre os níveis de sombreamento e as épocas após o transplantio, sendo aplicado o teste $\mathrm{F}$ a $95 \%$ de probabilidade e quando significativos, elaborados as curvas de regressão polinomial através do programa Sigma Plot ${ }^{\circledR}$ versão 11.0.

\section{RESULTADOS}

\subsection{Altura de plantas}

As mudas de jacarandá caviúna apresentaram maior incremento em altura (Figura 2A) a 5\% de significância, durante o período analisado, nos tratamentos com 70 e $84 \%$ de sombreamento atingindo maiores valores, com 153,33 e 143,33cm, respectivamente. A altura foi reduzida quando as mudas foram cultivadas sob baixos níveis de sombreamento.

As mudas de paineira apresentaram até os 90 DAE, um crescimento em altura similar em todos os tratamentos sendo observados maiores valores no tratamento com 50\% de sombra que atingiu um valor máximo de 135,74 cm nesta idade (Figura 3A). A altura das plantas apresentaram uma leve tendência a redução entre 120 e 140 DAE, porém o tratamento com 91\% de sombra nessa idade apresentou os maiores valores médios atingindo 191,33 cm de altura $(\mathrm{p}<0,05)$.

\subsection{Diâmetro do colo}

As mudas de jacarandá caviúna tiveram maiores diâmetro do colo $(\mathrm{p}<0,05)$ nos tratamentos intermediários de sombra (50\%, 70\% e $84 \%$ de sombra), sendo que todos os tratamentos apresentaram uma tendência de crescimento constante (Figura 2B).

Para as mudas de paineira os tratamentos que apresentaram os maiores diâmetros do colo foram os tratamentos com menores sombreamentos a ( $0 \%$ e 50\%) desde o início do crescimento, o que indica que as plantas não estiolaram. (Figura 3B).

\subsection{Relação altura/diâmetro}

Na análise da relação altura/ diâmetro verifica-se maior alocação para o crescimento em altura quando as mudas de jacarandá são cultivadas sob os maiores níveis de sombreamento (Figura 2C), sendo observados os maiores valores nos tratamentos com $84 \%$ e $91 \%$ atingindo maiores valores médios de 13,673 e 15,793, respectivamente, aos 180 DAE. Para as mudas de paineira também houve uma maior relação H/D nos tratamentos como maiores níveis de sombra (Figura 3C) apresentando valores médios iguais a 8,795 e 8,835 nos tratamentos com $84 \%$ e $91 \%$ respectivamente.

\section{4.Área foliar}

A área foliar do jacarandá caviúna somente foi afetada pelo sombreamento aos $90 \mathrm{DAE}(\mathrm{p}<0,05)$. Aos 180 DAE os valores médios observados foram superiores nos níveis de 70 e 84\% de sombra (3088,99 e 3357,06 $\mathrm{cm}^{2}$, respectivamente), sendo a menor área observada no tratamento com 0\% de sombra (Figura 2 D).

A área foliar das mudas de paineira, independente da idade, foi maior nos tratamentos com baixos níveis de sombra, sendo as maiores áreas observadas nos tratamentos com 0\% e 50\% de sombreamento aos 180 DAE (6179,75 e 6572,04 cm², respectivamente). Enquanto os tratamentos com maiores níveis de sombreamento proporcionaram menores áreas foliares (Figura 3D).

\section{DISCUSSÃO}

As mudas de jacarandá caviúna que cresceram sob 70 e $84 \%$ foram aquelas que apresentaram maiores alturas $(\mathrm{p}<0,05)$. Resultados semelhantes foram encontrados por Rêgo (2001) que verificou uma maior altura para essa espécie em sombreamentos de 64 e $70 \%$. Além desse outros autores analisando o crescimento de plantas lenhosas observaram um aumento na altura de mudas sombreadas (CHAVEZ; PAIVA, 2004; PAEZ et al., 2000; MAZZEI et al.,1999). Além disso, esta espécie tendeu a diminuir sua altura quando exposta a baixos níveis de sombra. Resultado também encontrado por outros autores para espécies lenhosas de crescimento tardio (MORAES NETO et al., 2000; SCALON et al., 2002).

A capacidade de crescer rapidamente em ambientes moderadamente sombreados é um mecanismo importante de adaptação da espécie e proporciona uma valiosa estratégia para escapar às condições de baixa intensidade luminosa (MORAES NETO et al., 2000). A adaptação em baixas intensidades luminosas é uma característica genética, a qual faz com que as folhas apresentem estrutura anatômica e propriedades fisiológicas que as capacitem ao uso efetivo da radiação solar disponível (LARCHER, 2006). 
Os resultados encontrados para o diâmetro do colo de jacarandá caviúna foram similares àqueles encontrados para altura, onde os maiores diâmetros foram observados nos tratamentos com níveis de sombreamento moderados. O maior crescimento em

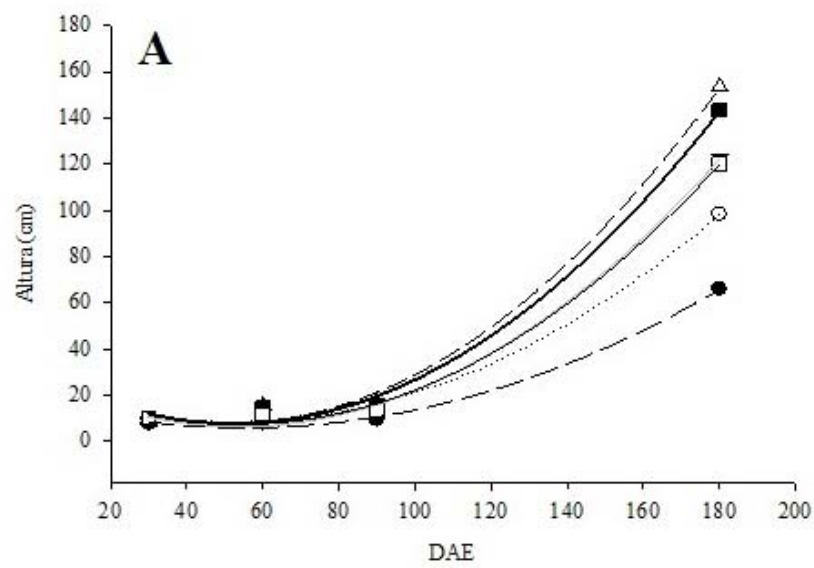

- $0 \% \quad Y=0,0038 x^{2}-0,4238 x+17,6121 / R^{2}=0,9978$

$22 \% \quad \mathrm{Y}=0,0055 \mathrm{x}^{2}-0,5751 \mathrm{x}+23,4365 / \mathrm{R}^{2}=0,994$

- $50 \%$ Y $=0,0073 x^{2}-0,7805 x+27,4061 / R^{2}=0,9960$

$70 \% \mathrm{Y}=0,0087 \mathrm{x}^{2}-0,8794 \mathrm{x}+30,1022 / \mathrm{R}^{2}=0,9950$

$84 \% \mathrm{Y}=0,0083 \mathrm{x}^{2}-0,8631 \mathrm{x}+30,3226 / \mathrm{R}^{2}=0,9948$

口 $91 \% \mathrm{Y}=0,0071 \mathrm{x}^{2}-0,7807 \mathrm{x}+28,7987 / \mathrm{R}^{2}=0,9967$

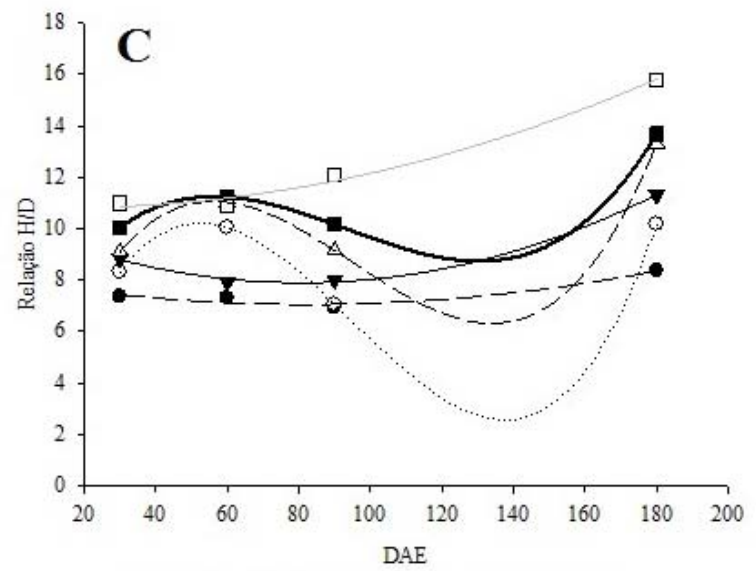

- $0 \% \quad \mathrm{Y}=0,0001 \mathrm{x}^{2}-0,0226 \mathrm{x}+7,9839 / \mathrm{R}^{2}=0,9993$

- $22 \% \quad Y=2,4840 E-005 x^{2}-0,0071 x^{2}+0,5381 x-2,1246 / R^{2}=0,9954$

- $50 \% \quad \mathrm{Y}=0,0003 \mathrm{x}^{2}-0,0562 \mathrm{x}+10,1633 / \mathrm{R}^{2}=0,9973$

$70 \% \mathrm{Y}=2,0179 \mathrm{E}-005 \mathrm{x}^{3}-0,0058 \mathrm{x}^{2}+0,4552 \mathrm{x}+0,0598 / \mathrm{R}^{2}=0,9987$

$84 \% \mathrm{Y}=1,2461 \mathrm{E}-005 \mathrm{x}^{2}-0,0035 \mathrm{x}^{2}+0,2750 \mathrm{x}+4,6067 / \mathrm{R}^{2}=9994$

$91 \% \quad \mathrm{Y}=0,0002 \mathrm{x}^{2}-0,0052 \mathrm{x}+10,8466 / \mathrm{R}^{2}=0,9997$ diâmetro do colo e altura podem indicar que a fotossíntese e a respiração encontram-se em um balanço favorável (DUTRA et al., 2012). Resultados semelhantes foram verificados para Goupia glabra (DANIEL et al., 1994), Caesalpinia echinata Lam.(AGUIAR et al., 2005) e

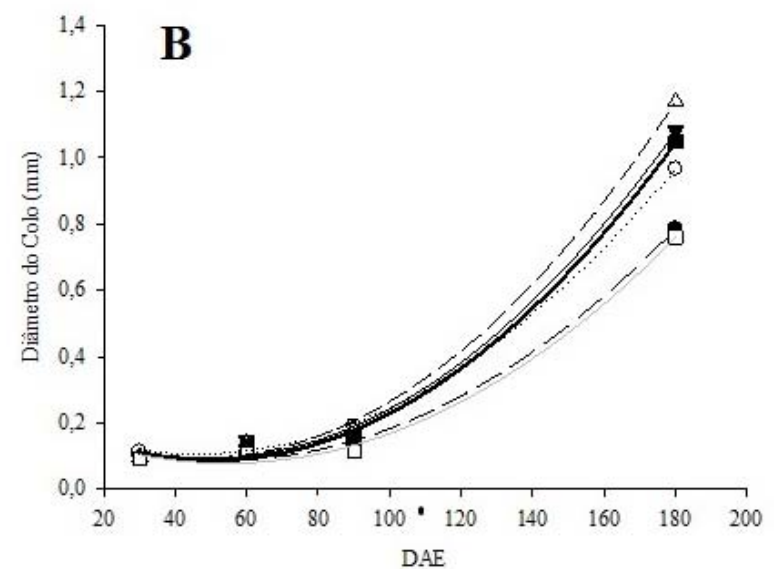

- $0 \% \quad \mathrm{Y}=4,3366 \mathrm{E}-005 \mathrm{x}^{2}-0,0046 \mathrm{x}+0,2099 / \mathrm{R}^{2}=0,9985$ $22 \% \mathrm{Y}=4,7909 \mathrm{E}-005 \mathrm{x}^{2}-0,0044 \mathrm{x}+0,2083 / \mathrm{R}^{2}=0,9995$

- $50 \% \mathrm{Y}=5,7700 \mathrm{E}-005 \mathrm{x}^{2}-0,0057 \mathrm{x}+0,2341 / \mathrm{R}^{2}=0,9952$

$\triangle 70 \% \mathrm{Y}=6,0696 \mathrm{E}-005 \mathrm{x}^{2}-0,0057 \mathrm{x}+0,2277 / \mathrm{R}^{2}=0,9976$

- $84 \% \mathrm{Y}=5,6658 \mathrm{E}-005 \mathrm{x}^{2}-0,0057 \mathrm{X}+0,2270 / \mathrm{R}^{2}=0,9962$

ㅁ $91 \% \mathrm{Y}=4,3180 \mathrm{E}-005 \mathrm{x}^{2}-0,0047 \mathrm{x}+0,2066 / \mathrm{R}^{2}=0,995$

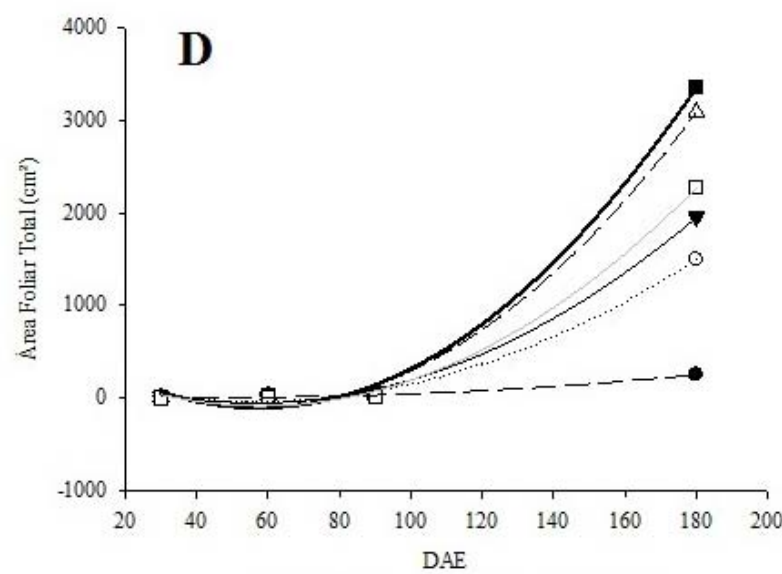

$0 \% \quad \mathrm{Y}=0,0144 \mathrm{x}^{2}-1,4441 \mathrm{x}+48,7747 / \mathrm{R}^{2}=0,9987$

$22 \% \mathrm{Y}=0,1003 \mathrm{x}^{2}-11,3534 \mathrm{x}+288,3197 / \mathrm{R}^{2}=0,9964$

T $50 \% \mathrm{Y}=0,1311 \mathrm{x}^{2}-14,8413 \mathrm{x}+370,6504 / \mathrm{R}^{2}=0,9972$

$\triangle 70 \% \mathrm{Y}=0,2096 \mathrm{x}^{2}-23,8914 \mathrm{x}+588,5598 / \mathrm{R}^{2}=0,9964$

- $84 \% \mathrm{Y}=0,2291 \mathrm{x}^{2}-26,2509 \mathrm{x}+651,7190 / \mathrm{R}^{2}=0,9967$

ㅁ $91 \% \mathrm{Y}=0,1576 \mathrm{x}^{2}-18,2900 \mathrm{x}+448,6735 / \mathrm{R}^{2}=0,9962$

Figura 2 - Crescimento inicial de Dalbergia nigra sobre diferentes níveis de sombreamento. (A) Crescimento em altura; (B) Diâmetro do colo; (C) relação H/D; Área foliar total; e DAE-Dias após a emergência.

Figure 2 - Initial growth of Dalbergia nigra at different levels of shading. (A) Height; (B) Diameter of the collar; (C) H/ $D$ ratio; (D) Total leaf area; and DAE-Days after emergence. 
Theobroma gradiflora (Willd ex Spreng) K.Schum (SILVA et al., 2007).

A área foliar das plantas de jacarandá caviúna foram maiores nos tratamento com 70 e $85 \%$ de sombra, diminuindo no tratamento com $0 \%$ de sombra. Este resultado corrobora com os resultados encontrados por Rêgo (2001) trabalhando com 34\%, 44\%, 64\% e $70 \%$ de sombra para essa mesma espécie. A diminuição da área foliar no tratamento com $0 \%$ de sombra pode
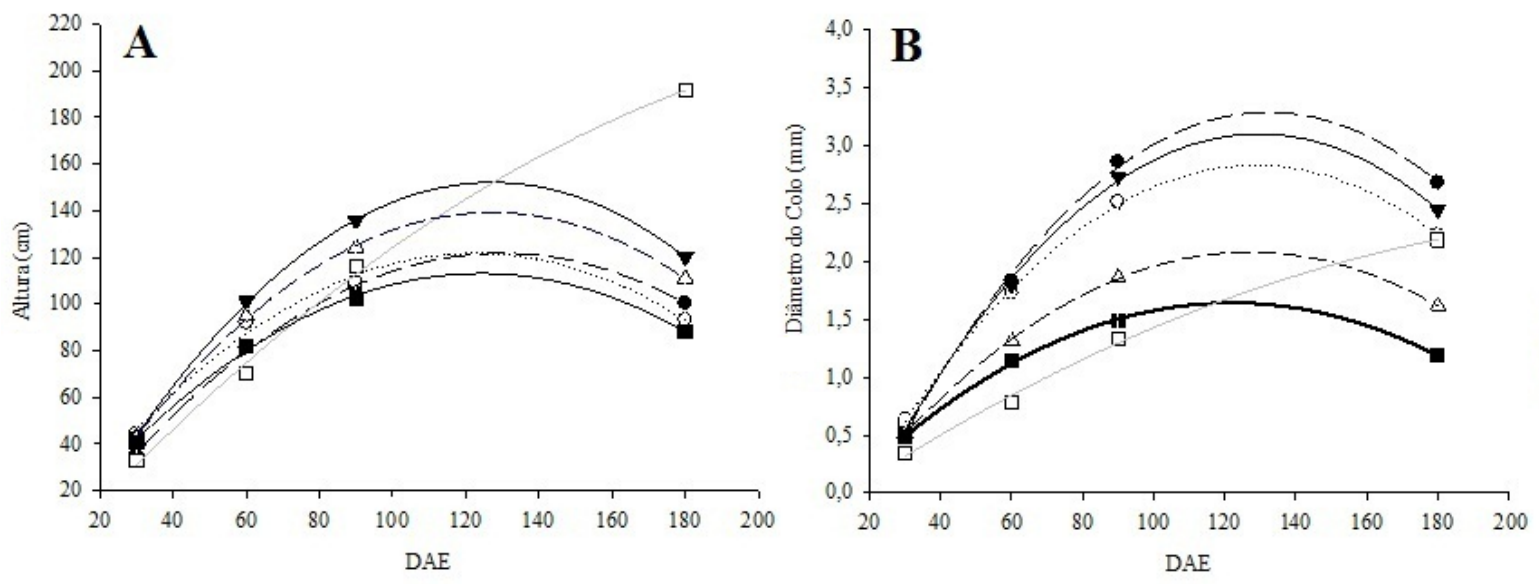

- $0 \% \quad \mathrm{Y}=-0,0086 \mathrm{x}^{2}+2,2379 \mathrm{x}-23,6301 / \mathrm{R}^{2}=0,9991$

$22 \% \mathrm{Y}=-0,0088 \mathrm{x}^{2}+2,1571 \mathrm{x}-10,5924 / \mathrm{R}^{2}=0,9848$

$50 \% \mathrm{Y}=-0,0115 \mathrm{x}^{2}+2,9213 \mathrm{x}-33,6275 / \mathrm{R}^{2}=0,9998$

$\triangle 70 \% \mathrm{Y}=-0,0102 \mathrm{x}^{2}+2,5846 \mathrm{x}-25,0133 / \mathrm{R}^{2}=0,9975$

- $84 \% \mathrm{Y}=-0,0033 \mathrm{x}^{2}+1,7550 \mathrm{x}-18,6803 / \mathrm{R}^{2}=0,9991$

ㅁ $91 \% \mathrm{Y}=-0,0080 \mathrm{x}^{2}+1,9932 \mathrm{x}-10,6883 / \mathrm{R}^{2}=0,996$

- $0 \% \quad \mathrm{Y}=-0,0003 \mathrm{x}^{2}+0,0703 \mathrm{x}-1,3609 / \mathrm{R}^{2}=0,9977$

$22 \% \mathrm{Y}=-0,0002 \mathrm{x}^{2}+0,0584 \mathrm{x}-0,9258 / \mathrm{R}^{2}=0,9991$

$50 \% \mathrm{Y}=0,0003 \mathrm{x}^{2}+0,0667 \mathrm{x}-1,2203 / \mathrm{R}^{2}=0,9982$

$\triangle 70 \% \mathrm{Y}=-0,0002 \mathrm{x}^{2}+0,0424 \mathrm{x}-0,6123 / \mathrm{R}^{2}=0,9997$

- $84 \% \mathrm{Y}=-0,0001 \mathrm{x}^{2}+0,0332 \times-0,3834 / \mathrm{R}^{2}=0,9988$

ㅁ $91 \% \mathrm{Y}=1,699 \mathrm{E}-005 \mathrm{x}^{2}+0,0214 \mathrm{x}-0,2880 / \mathrm{R}^{2}=0,9968$
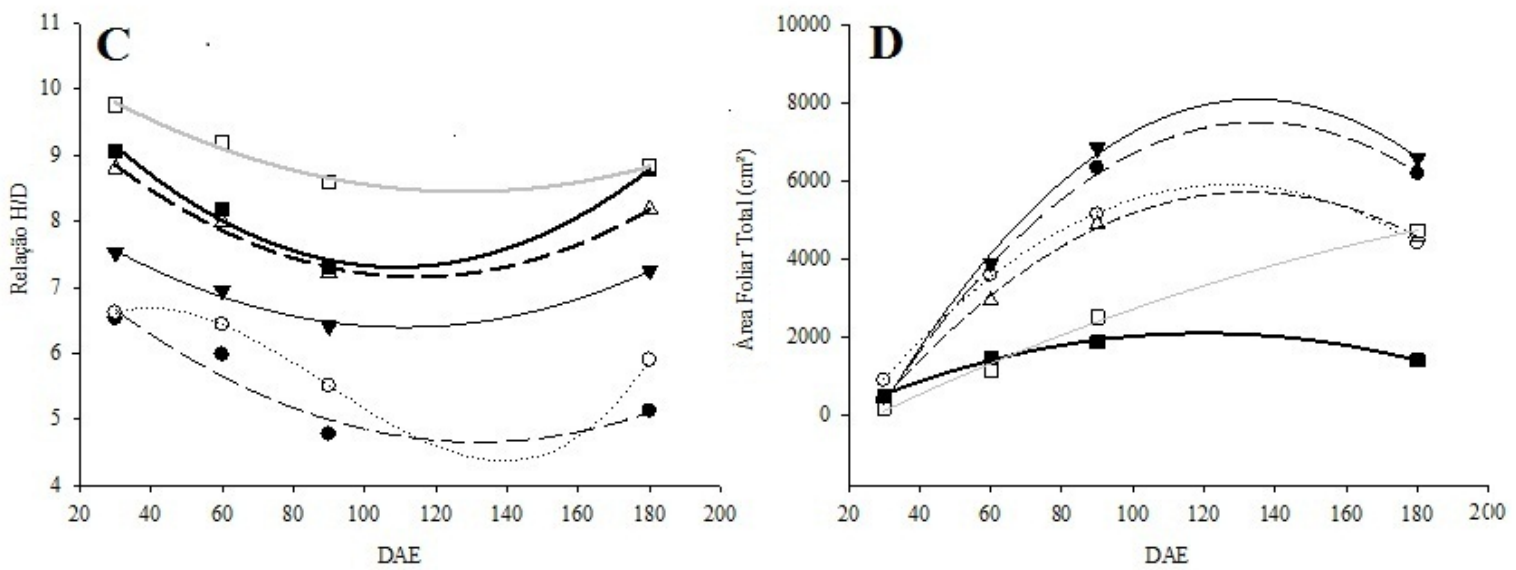

- $0 \% \quad Y=0,0002 x^{2}-0,0509 x+8,0111 / R^{2}=0,9158$

- $22 \% \quad Y=4,7544 E-006 x^{2}-0,0013 x^{2}+0,0790 x+5,2588 / R^{2}=0,9998$

- $50 \% \quad \mathrm{Y}=0,0002 \mathrm{x}^{2}-0,0395 \mathrm{x}+8,5818 / \mathrm{R}^{2}=0,9883$

$\triangle 70 \% \quad \mathrm{Y}=0,0002 \mathrm{x}^{2}-0,0541 \mathrm{x}+10,2562 / \mathrm{R}^{2}=0,9885$

- $84 \% \quad \mathrm{Y}=0,0003 \mathrm{x}^{2}-0,0639 \mathrm{x}+10,7892 / \mathrm{R}^{2}=0,9748$

ㅁ $91 \% \quad \mathrm{Y}=0,0001 \mathrm{x}^{2}-0,0360 \mathrm{x}+10,7497 / \mathrm{R}^{2}=0,9826$

- $0 \% \mathrm{Y}=-0,6490 \mathrm{x}^{2}+175,5084 \mathrm{x}-4368,1240 / \mathrm{R}^{2}=0,9959$

$22 \% \mathrm{Y}=-0,5320 \mathrm{x}^{2}+134,8894 \mathrm{x}-2653,5959 / \mathrm{R}^{2}=0,9993$

$50 \% \mathrm{Y}=-0,7177 \mathrm{x}^{2}+195,7928 \mathrm{x}-4861,5575 / \mathrm{R}^{2}=0,9963$

$\triangle 70 \% \mathrm{Y}=-0,5042 \mathrm{x}^{2}+133,6470 \mathrm{x}-3153,3820 / \mathrm{R}^{2}=0,9979$

- $84 \% \mathrm{Y}=-0,1939 \mathrm{x}^{2}+46,4857 \mathrm{x}-692,5243 / \mathrm{R}^{2}=0,9914$

$91 \% \mathrm{Y}=-0,0799 \mathrm{x}^{2}+47,624 \mathrm{x}-1252,4158 / \mathrm{R}^{2}=0,9960$

Figura 3- Crescimento inicial de Chorisia speciosa sobre diferentes níveis de sombreamento. (A) Crescimento em altura; (B) Diâmetro do colo; (C) relação H/D; Área foliar total; DAE-Dias após emergência.

Figure 3 - Initial growth of Chorisia speciosa at different levels of shading. (A)Heigth; (B) Diameter of the collar; (C) H/D Ratio; (D) Total leaf area; and DAE-Days after emergence.

Revista Árvore, Viçosa-MG, v.37, n.5, p.945-953, 2013 
está correlacionada com a maior luminosidade, maior temperatura e menor umidade que reduziram o crescimento das folhas. De acordo com Kitao et al. (2000) a exposição prolongada a altas irradiâncias pode ser prejudicial ás plantas, por absorverem mais luz do que podem utilizar, podendo ter como consequência a fotoinibição ou mesmo a morte da planta.

O aumento da área foliar, encontrado para as mudas de jacarandá, com o aumento do sombreamento é uma das maneiras da planta aumentar sua superfície fotossintetizante, assegurando maior aproveitamento de baixas intensidades luminosas, sendo tal comportamento próprio de espécies tolerantes à sombra (LARCHER, 2006). Os resultados encontrados neste estudo corroboram com a classificação de exigência a radiação proposta por Lorenzi (2002) para a espécie Dalbergia nigra, que a considera uma espécie secundária tardia a clímax, tolerante ao sombreamento leve a moderado na fase juvenil. Assim em condições naturais a espécie apresentaria melhores chances de regeneração sob a cobertura da mata, tolerando inclusive intensidades luminosas bastante reduzidas, em decorrência de um dossel fechado.

A altura de Chorisia especiosa foi superior no tratamento de $50 \%$ até os $90 \mathrm{DAE}$, onde foi superado pelo sombreamento de $90 \%$ até os 180 DAE. Estes resultados podem estar relacionados a uma necessidade de ambientes levemente iluminados nas fases iniciais do desenvolvimento e de acordo com Guariz et al. (2005) trabalhando com esta espécie obtiveram resultados semelhantes, sendo as maiores alturas observadas aos 150 dias nos tratamentos a $22 \%$ e $50 \%$ de sombreamento em comparação ao tratamento de pleno sol.

A capacidade de crescer rapidamente quando moderadamente sombreada é um mecanismo importante de adaptação da espécie. O alongamento do caule caracteriza uma resposta de evitação à sombra, em plantas consideradas heliófitas como a paineira, para maior captação da energia luminosa, indicando o envolvimento do fitocromo na percepção da sombra (TAIZ; ZEIGER, 2004).

O maior valor em altura observado no tratamento com $91 \%$ de sombra pode ter ocorrido em razão do estiolamento induzido pela baixa intensidade luminosa ou ainda pelas temperaturas mais amenas encontradas nesse tratamento que favoreceu o status hídrico da planta, de modo a permitir uma otimização da atividade fotossintética e da turgescência, necessária ao crescimento (REIS et al., 1992).

O diâmetro do colo para as mudas de paineira foi maior no tratamento sem sombreamento, resultados semelhantes foram observados para Ochroma lagopus e Brosimum rubescences, em condições de pleno sol até 30\% de sombreamento (CAMPOS; UCHIDA (2002); MARIMON et al., (2008). Os tratamentos com maior sombreamento apresentaram menores diâmetros e se, comparados com a altura, indicam o estiolamento das mudas em qualquer nível de sombreamento.

Assim constata-se que a espécie exige para o seu crescimento inicial uma maior quantidade de radiação, corroborando com a sua classificação como planta heliófita proposta por Lorenzi (2002) e quanto ao seu comportamento ecológico proposta por Nave et al. (1997) que a classifica desde pioneira a secundária tardia.

A área foliar da paineira foi maior nos tratamento com 0 e $50 \%$ de sombra, diminuindo nos tratamentos com elevado nível de sombreamento. O aumento da área foliar nas situações de pleno sol ( $0 \%$ de sombra) demonstra a capacidade de aumentar a sua superfície fotossintetizante, caracterizando a espécie como heliófita. Resultados similares foram observados por Scalon et al. (2001) trabalhando com mudas de pitangueira (Eugenia uniflora L.) sob condições de 50 e $70 \%$ de sombreamento e a pleno sol.

Assim em condições naturais, a paineira se beneficiaria com a presença de sunflecks e de clareiras no dossel. Além disso, suportaria as alterações nos níveis de luminosidades promovidas por grandes clareiras, principalmente em estágios mais tardios de seu crescimento (acima de $180 \mathrm{DAE}$ ).

Ambas espécies tiveram um aumento na relação H/D nos tratamentos com maiores níveis de sombreamento, corroborando com as respostas ecológicas encontradas para essas espécies nesse trabalho. A relação H/D pode ser utilizada para identificar a qualidade da muda, pois plantas com baixo diâmetro do colo apresentam dificuldades de se manterem eretas após o plantio. Uma relação adequada entre esses parâmetros permite uma maior taxa de sobrevivência em campo e melhor desenvolvimento após o plantio (VIANA et al., 2008).

Carneiro (1995) afirma que as relações H/D devem situar-se entre os limites de 5,4 até 8,1 em qualquer 
fase do período de produção de mudas. Desta forma os maiores valores encontrados para ambas espécies nos tratamentos com maiores níveis de sombreamento situam-se acima desse limite estabelecido, não apresentando mudas de qualidade nessas condições, indicando que essas devem ser cultivadas em sombreamentos mais leves e plantadas no campo antes do 180 DAE.

\section{CONCLUSÕES}

A espécie Dalbergia nigra se comportou tolerante a sombra e com estágios mais avançados de sucessão se aproxima de espécies secundárias tardias a clímax.

A espécie Chorisia speciosa apresentou características de espécies heliófitas estando presente em etapas iniciais de sucessão, aproximando-se de plantas pioneiras a secundárias iniciais.

\section{AGRADECIMENTOS}

Os autores agradecem à Fundação de Amparo a Pesquisa do Estado do Rio de Janeiro e à Coordenadoria de Aperfeiçoamento de Pessoal de Nível Superior pelo auxílio financeiro para realização do trabalho.

\section{REFERÊNCIAS}

AGUIAR, F. F. A. et al. Germinação de sementes e formação de mudas de Caesalpinia echinata Lam. (Pau-Brasil): Efeito do Sombreamento I. Revista Árvore, v.29, n.6, p.871-875, 2005.

AZEVEDO, I. M. G. et al. Estudo do crescimento e qualidade de mudas de marupá (Simarouba amara Aubl.) em viveiro. Acta Amazonica, v.40, n.1, p.157-164, 2010.

CAMPOS, M. A. A.; UCHIDA, T. Influência do sombreamento no crescimento de mudas de três espécies amazônicas. Pesquisa

Agropecuária Brasileira, v.37, n.3, p.281288, 2002.

CARneiro, J. G. A. Produção e controle de qualidade de mudas florestais. Viçosa, MG: Folha de Viçosa, 1995. p.63-95.

CARVAlho, P. E. R. Espécies Arbóreas Brasileiras. Brasília: Embrapa-CNPF, 2003.

Revista Árvore, Viçosa-MG, v.37, n.5, p.945-953, 2013
CHAVES, A. S.; PAIVA, H. N. Influência de diferentes períodos de sombreamento sobre a qualidade de mudas de fedegoso (Senna macranthera (Collad). Irwin et Barn.). Scientia Florestalis, v.65, n.1, p.22-29, 2004.

DANIEL, O.; OHASHI, S. T.; SANTOS, R. A. Produção de mudas de Goupia glabra (cupiúba) efeito dos níveis de sombreamento e tamanhos de embalagens. Revista Árvore, v.18, n.1, p.1-13, 1994.

DUTRA, T. R. et al. Desenvolvimento inicial de mudas de copaíba sob diferentes níveis de sombreamento e substratos. Revista

Ciência Agronômica, v.43, n.2, p.321-329, 2012.

FANTI, S. C.; PEREZ, S. C. J. G. A. Influência do sombreamento artificial e da adubação química na produção de mudas de Adenanthera pavonina L. Ciência Florestal, v.13, n.1, p.49-56, 2003.

GUARIZ, H. R. et al. Crescimento de mudas de paineira (Chorisia speciosa $\mathrm{St}$. Hil) submetidas a diferentes níveis de sombreamento. In: ENCONTRO LATINO AMERICANO DE INICIAÇÃO CIENTÍFICA, 10/ENCONTRO LATINO AMERICANO DE PÓS-GRADUAÇÃO, 6., 2005, Alegre. Anais... Alegre: 2005. p.2862-2864.

LIMA JUNIOR, E. C. et al. Trocas gasosas, características das folhas e crescimento de plantas jovens de Cupania vernalis Camb. submetidas a diferentes níveis de sombreamento. Ciência Rural, v.35, n.5, p.1092-1097, 2005.

KITAO, M. et al. Temperature response and photoinhibition investigated by chlorophyll fluorescence measurements for four distinct species of dipterocarp trees. Physiologia Plantarum, v.109, n.3, p.284-290, 2000.

LARCHER, W. Ecofisiologia vegetal. São Carlos; Rima, 2006. p.550.

LORENZI, H. Árvores Brasileiras: manual de identificação e cultivo de plantas arbóreas nativas do Brasil. 4 ed. Nova Odessa: Plantarum, 2002. v.1. p.368. 
MARIMON, B. S. et al. Desenvolvimento inicial e partição de biomassa de Brosimum rubescens Taub. (Moraceae) sob diferentes níveis de sombreamento. Acta Botanica Brasilica, v.22, n.4, p.941-953, 2008.

MAZZEI, L. J. et al. Crescimento de plântulas de Hymenaea courbaril var. stilbocarpa (Hayne)

Lee \& Lang em viveiro. Boletim do Herbário Ezechias Paulo Heringer, v.21, n.4, p.21-29, 1999.

MORAES NETO, S.P. et al. Crescimento de mudas de algumas espécies arbóreas que ocorrem na Mata Atlântica, em função do nível de luminosidade. Revista Árvore, v.24, n.1, p.3545, 2000.

NAVE, A. G.; RODRIGUES, R. R.; GANDOLFI, S. Planejamento e recuperação ambiental da fazenda de São Pedro da Mata, Município de Riolândia, SP. In: SIMPÓSIO NACIONAL DE RECUPERAÇÃO DE ÁREAS DEGRADADAS, 3., "Do substrato ao solo: trabalhos voluntários”, 1997, Ouro Preto. Anais... Ouro Preto: 1997.p.67-77.

OLIVEIRA, A. K. M.; PEREZ, S. C. J. G.A. Crescimento inicial de Tabebuia aurea sob três intensidades luminosas. Ciência Florestal, v.22, n.2, p.263-273, 2012.

OLIVEIRA FILHO, A. T. Estudos ecológicos da vegetação como subsídios para programa de revegetação com espécies nativas: uma proposta metodológica. Cerne, v.1, n.1, p.113-117, 1994.

ORTEGA, A. R. et al. Avaliação do crescimento de mudas de Psidium cattleianum Sabine a diferentes níveis de sombreamento em viveiro. Cerne, v.12, n.3, p.300-308, 2006.
PAEZ, A. et al. Growth, soluble carbohydrates, and aloin concentration of Aloe vera plants exposed to three irradiance levels.

Environmental and Experimental Botany, v.44, n.1, p.133-139, 2000.

REGO, G. M. Ecofisiologia do jequitibárosa e do jacarandá-da-Bahia: morfogênese, germinação e crescimento inicial. 2001. 84f. Tese (Doutorado em Produção Vegetal)Universidade Federal do Paraná, Curitiba, 2001.

REIS, A. et al. Aspectos sobre a Conservação da Biodiversidade e o Manejo da Floresta Tropical Atlântica. Revista do Instituto Florestal, v.4, n.4, p.169-173, 1992.

SCALON, S. P. Q. et al. Germinação e crescimento de mudas de pitangueira (Eugenia uniflora L.) sob condições de sombreamento. Revista Brasileira de Fruticultura, v.23, n.3, p.652-655, 2001.

SCALON, S. P. Q. et al. Crescimento inicial de mudas de espécies florestais nativas sob diferentes níveis de sombreamento. Revista Árvore, v.26, n.1, p.1-5, 2002.

SILVA, R. R. et al. Desenvolvimento inicial de plântulas de Theobroma grandiforum (Willd.ex Spreng.) Schum. sob influência de sombreamento. Acta Amazonica, v.37, n.3, p.365-370, 2007.

TAIZ, L.; ZEIGER, E. Fisiologia vegetal. 3.ed. Porto Alegre: Artmed, 2004. 719p.

VIANA, J. S. et al. Crescimento de mudas de Bauhinia forficata Link. em diferentes tamanhos de recipientes. Floresta, v.38, n.4, p.663-671, 2008. 
